DR. TIM OUTHRED (Orcid ID : 0000-0003-2534-3635)

MS. GRACE MORRIS (Orcid ID : 0000-0003-3598-9712)
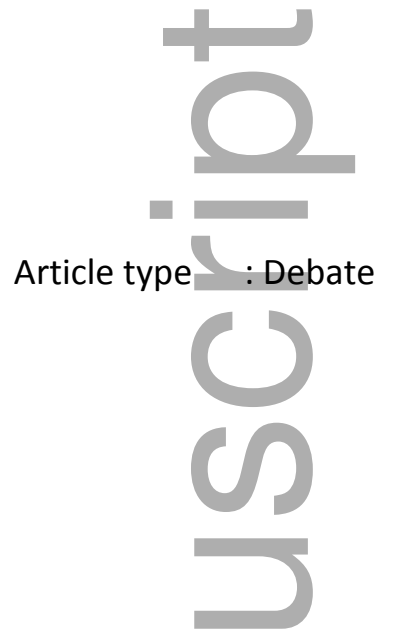

Debate

\title{
Defining disorders with permeable borders: You say bipolar, I say borderline!
}

Darryl Bassett ${ }^{1,2}$, Roger Mulder ${ }^{1,3}$, Tim Outhred ${ }^{1,4,5,6}$, Amber Hamilton $^{1,4,5,6}$, Grace Morris ${ }^{1,4,5,6}$, Pritha Das $^{1,4,5,6}$, Michael Berk ${ }^{1,7,8}$, Bernhard T Baune ${ }^{1,9}$, Philip Boyce ${ }^{1,10}$, Bill Lyndon ${ }^{1,5,11,12}$, Gordon Parker ${ }^{1,13,14}$, Ajeet B Singh ${ }^{1,15}$, Gin S Malhi ${ }^{1,4,5,6}$.

${ }^{1}$ Mood Assessment and Classification Committee (MAC Committee). See Footnote.

${ }^{2}$ Private practice in Psychiatry and Division of Psychiatry, University of Western Australia

${ }^{3}$ Department of Psychological Medicine, University of Otago - Christchurch, Christchurch, New Zealand

${ }^{4}$ Academic Department of Psychiatry, Northern Sydney Local Health District, St Leonards, NSW, Australia

${ }^{5}$ Sydney Medical School Northern, University of Sydney, Sydney, NSW, Australia

${ }^{6}$ CADE Clinic, Royal North Shore Hospital, Northern Sydney Local Health District, St Leonards, NSW,

Australia

${ }^{7}$ Deakin University, School of Medicine, IMPACT Strategic Research Centre, Melbourne, VIC, Australia

${ }^{8}$ Department of Psychiatry, Orygen Research Centre, and the Florey Institute for Neuroscience and

Mental Health, University of Melbourne, Melbourne, VIC, Australia

${ }^{9}$ Discipline of Psychiatry, University of Adelaide, Adelaide, South Australia, Australia

This is the author manuscript accepted for publication and has undergone full peer review but has not been through the copyediting, typesetting, pagination and proofreading process, which may lead to differences between this version and the Version of Record. Please cite this article as doi: $\underline{10.1111 / \text { bdi. } 12528}$

This article is protected by copyright. All rights reserved 
${ }^{10}$ Discipline of Psychiatry, Sydney Medical School, Westmead Clinical School, University of Sydney, Sydney, NSW, Australia

${ }^{11}$ Mood Disorders Unit, Northside Clinic, Greenwich, NSW, Australia

${ }^{12}$ ECT Services Northside Group Hospitals, Greenwich, NSW, Australia

${ }^{13}$ School of Psychiatry, University of New South Wales

${ }^{14}$ Black Dog Institute, Sydney, New South Wales, Australia

${ }^{15}$ School of Medicine, IMPACT Strategic Research Centre, Deakin University, Geelong, VIC, Australia

Correspondence: MACC Chair. CADE Clinic, Academic Department, Level 3, Main Building, RNSH, St Leonards, Sydney, NSW 2065, Australia. Email: gin.malhi@sydney.edu.au

Footnote: The Mood Assessment and Classification Committee (MAC Committee) comprised academic psychiatrists with clinical expertise in the management of mood disorders and researchers with an interest in depression and bipolar disorders. The independently convened committee specifically targeted contentious aspects of mood disorders diagnosis and assessment with the express aim of informing clinical practice and future research. Members of the committee held one face to face meeting in Sydney (Australia) to discuss the issues in depth and agree upon outcomes. These were then developed further via email correspondence.

The interface between Borderline Personality Disorder (BPD) and Bipolar Disorders (BDs) has been the subject of much clinical and academic debate. Principally because the conditions share many clinical and presumably biological characteristics and the confluence has important treatment and risk assessment implications ${ }^{1,2}$.

Depressive signs and symptoms are almost ubiquitous in both ${ }^{3}$, creating a strong association between BPD and mood disorders to the extent that some have suggested that BPD should be reclassified as a mood disorder ${ }^{4}$, specifically a condition of recurrent unstable mood and behaviour that could even be given an alternative name, fluoxothymia. However, others have countered that BPD is a complex and multidimensional syndrome whose symptoms are not confined to changes in mood ${ }^{1,2}$, and while there is no clear qualitative distinction between BPD 
and BDs, meaningful differences can be made with the current weight of evidence suggesting that BPD and BDs are fundamentally separate entities.

\section{Comparison of Clinical Features}

Determining a diagnosis of BPD in contrast to BDs is best achieved by adopting a longitudinal and developmental perspective. In addition, some cross-sectional features can be useful (see Fig 1, Panel A). Provisional diagnoses of BPD or BDs are mostly accompanied by corresponding diagnoses in first-degree family members, if either disorder is present. BPD patients present with pervasive evidence of a "noxious sense of self"1, profoundly negative cognitive schemata, negative emotional evaluations of themselves as people, and marked difficulty perceiving interpersonal relationships as persistently trustworthy and positive. In contrast BD patients tend to have similar (or only mildly negative) pervasive perceptions of self and interpersonal relationships, compared with the healthy population. Indeed the pervasive and recurring severe disruption of interpersonal relationships for the patient with BPD is a major (but not exclusive) distinguishing feature. A corollary of this is the observation that, in relationships with patients suffering BPD (including very brief contacts), other people frequently experience intense and personally meaningful negative emotions, described by psychoanalysts as "projective identification". This process is rarely observed in BD and is often clinically helpful as further confirmation of a diagnosis of BPD. The interpersonal consequences of BD emerge in the context of mood syndrome related behavioural issues, the forerunner of which is often found in a developmental history of childhood maltreatment - which weights diagnosis more to BPD than BDs.

Confusingly, emotional dysregulation is a major feature of both BPD and BDs, but in BPD it tends to be rapid, with changes lasting hours to days and closely linked to interpersonal events, while in BDs the profile is extended and stable over days to months (with the exception of mixed affective states). In addition, in BPD the emotional shifts are mostly between depression and rage, but between depression and mania in BDs (except in mixed affective states or during the frustration of manic behaviour, where rage is more common). The element of rage that 
pervades BPD affective dysregulation has been referred to as an 'affective storm' that can mimic mixed states.

Attempted suicide is more common in BPD (while suicide is of similar frequency) and a history of disruption to attachments such as sexual abuse and other early trauma is significantly more likely in BPD than BDs - a key aspect that has to be discerned when collating a developmental history. Sleep disturbance also occurs in both groups of disorders, but more often has specific characteristics in BDs, such as hypersomnia in bipolar depression and a reduced need for sleep in mania. Impulsivity is, again, common to both, but more pervasive in BPD as it is limited to the elevated phase of illness in BDs. Psychosis may occur in BPD, but is generally very limited in content and duration (hours or days), while psychosis in BDs (when it occurs) is usually prominent and persistent for days or weeks. Interestingly, in BPD dissociation appears to be the mechanism that underpins psychotic symptoms, and awareness of this is important because misdiagnosis can lead to inappropriate pharmacotherapy.

\section{Clinical Approach}

In cases where the diagnosis is initially unclear and distress is high, the clinical presentation may improve with an indicated treatment (see Fig 1, Panel B). BDs usually respond very well to medication such as mood stabilizers and atypical antipsychotics and ECT, whereas the benefits for BPD are much more limited. However bipolar depression often does not respond to antidepressants and this is also observed in BPD (unless accompanied by melancholic depression). Going deeper, differential responses to antidepressants (e.g., partial or nonresponse, treatment-emergent affective switch) may also be indicative. BPD does not respond to ECT, in contrast to BDs. Finally, psychological therapies are the mainstay of management for BPD, though for the treatment of acute BD depression and prophylaxis in psychological therapies have proven to be of significant benefit. In this way, patients can be trialled on treatments in the short-to-medium term to better differentiate the disorders, in an attempt to determine a suitable treatment approach and ameliorate distress as quickly as possible.

Given the overlapping nature and complexity of symptoms that present cross-sectionally, longitudinal observation is invaluable, as the patterns of self-perception and evaluation, 
interpersonal relationships, symptoms and response to treatment will be most accurately identified and evaluated over a period of months or years, rather than weeks or months. The developmental histories of BPD (characterised by dysfunctional attachment) those with BPD and BDs also differ and this can only be established over decades of life experience. BPD tends to first become evident around puberty and BDs first evident in late adolescence or early adulthood. BPD tends to become less florid in symptomatology by the fourth and fifth decades, with patients experiencing less distress (including an improved sense of self, less rage and less self-destructiveness) and building relationships with greater satisfaction. BDs, in contrast, tends to persist and even become more severe and disabling (particularly depressive symptoms) with advancing age, with the age-related shift being from more mania in youth to more depression with age.

The existence of biomarkers to aid in distinguishing between BPD and BDs would be helpful, and our knowledge of the neurobiology of these disorders has advanced considerably over time. The processes of the fronto-limbic network have been the prime focus of attention and Malhi et al..$^{5}$ reported evidence that BPD was associated with reduced feedback to the prefrontal cortex from the amygdala (less "bottom-up feedback") and BDs with reduced regulation of emotion by the dorsolateral pre-frontal cortex (reduced "top-down cognitive processing"). While at the moment the methodology required for such psychophysiological assessment is beyond clinical practice, the findings from such research offer potential for the future if they can prove sensitive, specific, and cost-effective enough for clinical translation.

\section{Conclusion}

In summary, diagnostic distinctions between BPD and BDs (and mood disorders generally) do exist, although these disorders have a 'semi-permeable' boundary. A combination of crosssectional and longitudinal assessment (including developmental history of childhood maltreatment) is required to provide optimal confidence in the diagnosis achieved, appropriateness of care, and validity of risk assessment while recognising that the two conditions can co-exist. 
Acknowledgement: The MAC Project was supported logistically by Servier who provided financial assistance with travel and accommodation for those MAC Committee members travelling interstate or overseas to attend the meeting in Sydney (held on $18^{\text {th }}$ March 2017). Members of the committee were not paid to participate in this project and Servier had no input into the content, format or outputs from this project.

\section{References}

1. Bassett D. Borderline personality disorder and bipolar affective disorder: Spectra or spectre? A review. Australian \& New Zealand Journal of Psychiatry. 2012; 46(4):327-29.

2. Paris J, Black DW. Borderline personality disorder and bipolar disorder: What is the difference and why does it matter? Journal of Nervous and Mental Disease. 2015; 203(1):3-7.

3. Tyrer P. Why borderline personality disorder is neither borderline nor a personality disorder. Personality and Mental Health. 2009; 3(2): 86-95.

4. Zimmerman M, Martinez, JH, Morgan TA, Young D, Chelminski I, Dalrymple K. Distinguishing bipolar II depression from major depressive disorder with comorbid borderline personality disorder: demographic, clinical, and family history differences. The Journal of Clinical Psychiatry. 2013; 74(9):880-886.

5. Malhi GS, Tanious M, Fritz K, Coulston CM, Bargh DM, Phan KL, Calhoun V, Das P. Differential engagement of the fronto-limbic network during emotion processing distinguishes bipolar and borderline personality disorder. Molecular Psychiatry. 2013; 18(12): 1247-1248.

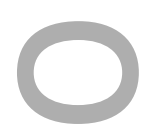

Fig 1. An illustration of the disorder characteristics and biological treatment efficacy in BPD and BD.

Panel A. Depicts the relative prominence of the risk factors and cross-sectional features in BPD and BD. In terms of aetiology, sexual abuse and early trauma tends to be prominent in BPD. Key cross-sectional features that tend to differentiate the disorders-based on their prominence-are self and relational dysfunctions, and sleep disturbance in BD depression and mania. Equally prominent-yet qualitatively different-features are affective/mood dysregulation (hours-days in BPD; days-months in BD), impulsivity (generally in BPD; mostly in mania for BD), suicide (though attempts are more prominent in BPD), and psychosis/dissociative symptoms (dissociative symptoms common in BPD; psychosis in extreme cases of BD depression and mania). 
Panel B. Depicts the efficacy of treatments across BPD and BD. Given that BD has different treatment phases (mania, depression, prophylaxis), these are differentiated. In general, biological treatments are more effective for managing $B D$, whereas response rates tend to be relatively modest in BPD across all classes of agents. In contrast, psychological therapies are better suited in the treatment of BPD especially in maintaining wellness. In this way, BPD and BD can be separated according to treatment efficacy.

$\square$
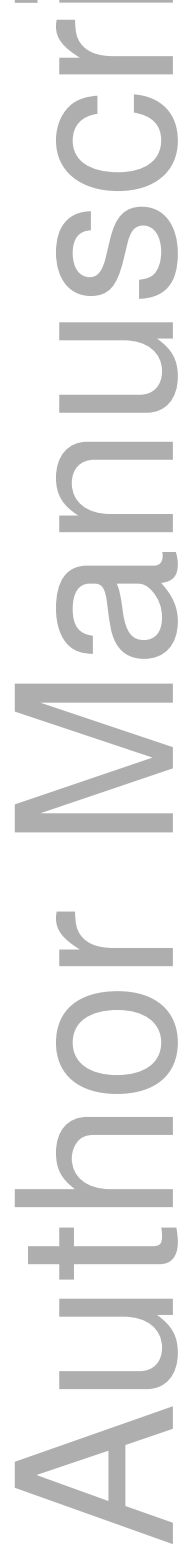

This article is protected by copyright. All rights reserved 


\section{A. Relative prominence of factors and features in BPD and BD}

Risk factors

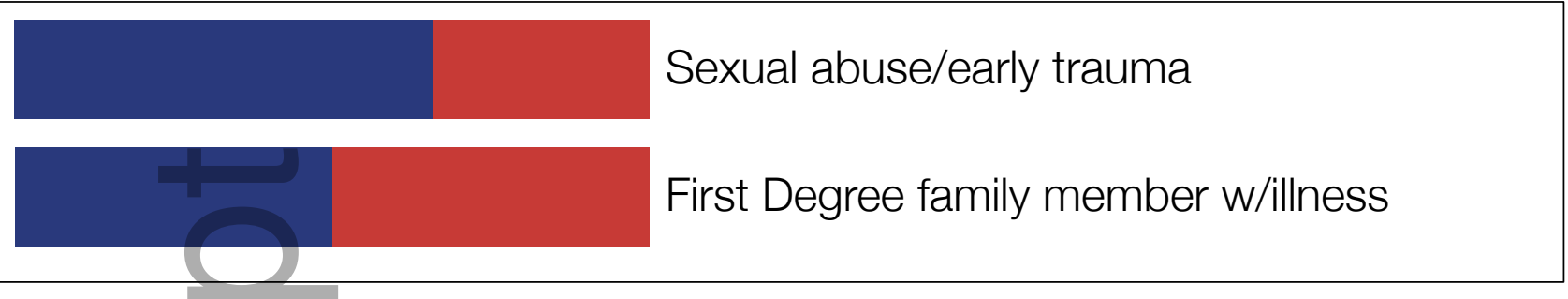

Cross-sectional features

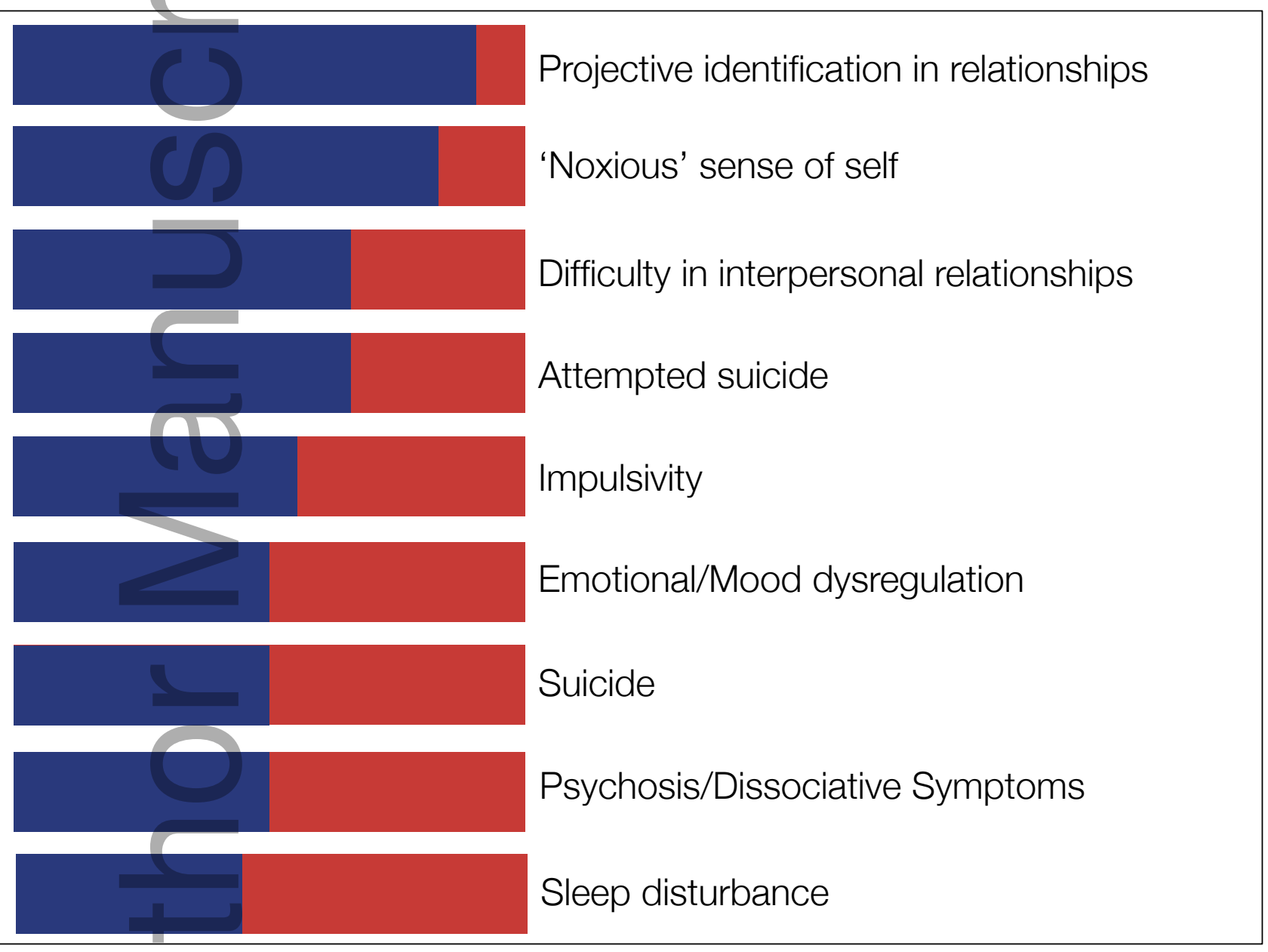

\section{B. Efficacy of treatments across BPD and BD}

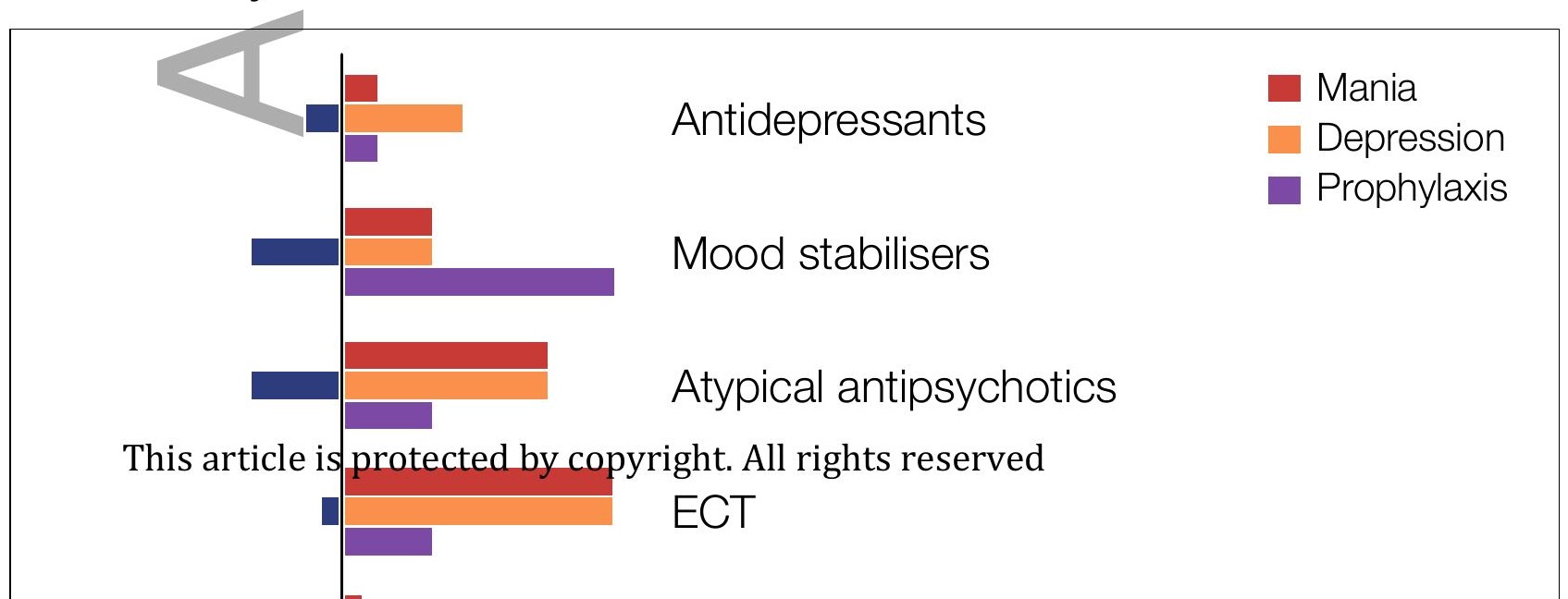




\section{University Library}

\section{$\bullet$ M I N E R VA}

\section{A gateway to Melbourne's research publications}

Minerva Access is the Institutional Repository of The University of Melbourne

\section{Author/s:}

Bassett, D;Mulder, R;Outhred, T;Hamilton, A;Morris, G;Das, P;Berk, M;Baune, BT;Boyce, P;Lyndon, B;Parker, G;Singh, AB;Malhi, GS

Title:

Defining disorders with permeable borders: you say bipolar, I say borderline!

\section{Date:}

2017-08-01

\section{Citation:}

Bassett, D., Mulder, R., Outhred, T., Hamilton, A., Morris, G., Das, P., Berk, M., Baune, B. T., Boyce, P., Lyndon, B., Parker, G., Singh, A. B. \& Malhi, G. S. (2017). Defining disorders with permeable borders: you say bipolar, I say borderline!. BIPOLAR DISORDERS, 19 (5), pp.320-323. https://doi.org/10.1111/bdi.12528.

Persistent Link:

http://hdl.handle.net/11343/293260 